\title{
A general-purpose hierarchical mesh partitioning method with node balancing strategies for large-scale numerical simulations
}

\author{
Fande Kong ${ }^{\dagger *}$, Roy H. Stogner ${ }^{\ddagger}$, Derek R. Gaston ${ }^{\dagger}$, John W. Peterson ${ }^{\dagger}$, Cody J. Permann ${ }^{\dagger}$, \\ Andrew E. Slaughter ${ }^{\dagger}$, and Richard C. Martineau ${ }^{\dagger}$ \\ ${ }^{\dagger}$ Department of Modeling and Simulation \\ Idaho National Laboratory, Idaho Falls, Idaho, USA \\ ${ }_{\ddagger}^{\ddagger}$ Institute for Computational and Engineering Sciences \\ The University of Texas, Austin, Texas, USA \\ *Corresponding Author: fande.kong@inl.gov
}

\begin{abstract}
Large-scale parallel numerical simulations are essential for a wide range of engineering problems that involve complex, coupled physical processes interacting across a broad range of spatial and temporal scales. The data structures involved in such simulations (meshes, sparse matrices, etc.) are frequently represented as graphs, and these graphs must be optimally partitioned across the available computational resources in order for the underlying calculations to scale efficiently. Partitions which minimize the number of graph edges that are cut (edgecuts) while simultaneously maintaining a balance in the amount of work (i.e. graph nodes) assigned to each processor core are desirable, and the performance of most existing partitioning software begins to degrade in this metric for partitions with more than than $O\left(10^{3}\right)$ processor cores. In this work, we consider a general-purpose hierarchical partitioner which takes into account the existence of multiple processor cores and shared memory in a compute node while partitioning a graph into an arbitrary number of subgraphs. We demonstrate that our algorithms significantly improve the preconditioning efficiency and overall performance of realistic numerical simulations running on up to 32,768 processor cores with nearly $10^{9}$ unknowns.
\end{abstract}

Index Terms-graph partitioning, parallel numerical simulation, finite element method, load balance, parallel scalability, scientific computing

\section{INTRODUCTION}

As the compute node density, complexity, and heterogeneity of modern supercomputers continues to advance, so too does the potential for parallel numerical simulations to tackle ever more detailed and demanding physical applications, and the need for sophisticated numerical algorithms which are tailored to the underlying hardware. The manner in which the data structures and workload of a given simulation are partitioned among the available computational resources plays a critical role in the overall parallel efficiency which can be achieved, and the need to determine an "optimal" partitioning becomes even more acute as the number of processor cores gets large.

In this work, we focus on developing a partitioning algorithm for subdividing a large-scale graph (corresponding, e.g., to the dual graph of a finite element mesh) into $n p$ subgraphs of nearly equal size, while minimizing the number of edge-cuts required to do so. In this context, $n p$ is the number of processor cores, the size of a processor's subgraph is proportional to its computational workload (thus, producing equal size subgraphs is sometimes referred to as "load balancing"), and the number of edge-cuts corresponds to the amount of inter-process communication required to share information between parts of the graph. Computational workload calculations can also be generalized to include the effects of so-called "weighted" graphs. In a weighted graph, each vertex (and possibly each edge) is assigned a "weight" value according to some metric, and the corresponding workload is computed by summing the weights. The algorithm proposed in this work supports weighted graphs, but no examples are considered here.

The computation of an optimal partitioning is known to be an NP-complete problem [1], so heuristics and approximations must be employed to find acceptable partitions in a reasonable time [2]. Interfaces between adjacent partitions result in shared degrees of freedom (DOFs) which are communicated among neighboring partitions during various parts of the simulation such as solver and preconditioner applications. Parallel scalability of solvers and preconditioners is a fundamental requirement for constructing an efficient simulation capability, and this scalability depends directly on partition quality. Poor-quality partitions can lead to workload imbalances and unnecessary communication, two major causes of parallel inefficiency.

Partitioning is an active research topic, and many approaches, including geometric [3], graph-based [4], hierarchical [5], and spectral methods [6] have been proposed, studied, and implemented in numerical software packages. For larger processor counts, multilevel partitioning frameworks [7] have demonstrated reasonably good results. The typical multilevel method consists of three stages: coarsening, partitioning, and refinement. First, the original large-scale graph is coarsened several times to reduce the graph size. Next, the coarsest graph undergoes partitioning (typically using a spectral method or graph-based algorithm). Finally, the partition is improved during a refinement procedure, often using the local opti- 
mization algorithms of Kernighan-Lin [8] (KL) and FiducciaMattheyses [9] (FM).

Based on the multilevel framework, several successful general-purpose serial tools for graph partitioning have been developed, including CHACO [10], METIS [11] and Scotch [12]. Most graphs of interest in large-scale numerical simulations are too large to be partitioned on a single processor core, so distributed memory parallel graph partitioning tools have also been developed, including ParMETIS [13] and PTScotch [12], which are based on underlying serial multilevel partitioners.

Existing serial and parallel partitioners work well for up to $O\left(10^{3}\right)$ processor cores, but they may be far from ideal or even fail to generate partitions for larger numbers of processor cores. Existing partitioners also frequently don't account for the layout of the processing cores on multi-core systems by assigning neighboring partitions to cores on the same compute node, an approach that can benefit from memory locality and avoiding off-node communications entirely.

To address this shortcoming, we develop a hierarchical partitioning approach which accounts for the existence of multiple cores per compute node which is standard on modern supercomputers. A similar idea has also been discussed in our previous work [14]-[18].

In the present study, the hierarchical partitioning approach is extended to more general cases involving an arbitrary number of submeshes, while still taking into account the number of cores per compute node and the shared memory configuration. If the partitioner is run as a preprocessing step, the proposed algorithm can use a small number of processor cores to partition a graph into a large number of subgraphs. In addition, our new algorithm ensures that the shared mesh nodes are evenly distributed among the neighboring processor cores, thereby improving load balancing and ultimately the overall parallel efficiency of the simulation. The new algorithm is referred to as the "general-purpose hierarchical mesh partitioning approach" and abbreviated herein as "Hierarch".

The rest of this paper is organized as follows. In Section 2 , the general-purpose hierarchical mesh partitioning approach is described in detail. The various node assignment strategies are described in Section 3, and numerical results with up to 32,768 processor cores are given in Section 4. The results are summarized and conclusions are drawn in Section 5.

\section{HieRARCHICAL MESH PARTITIONING}

To further motivate the need for optimal partitioning and the consequences of a poor partition, we first consider the simple 2D example shown in Fig. 1. In this example, a mesh is distributed across 8 different processors using two different partitioning algorithms, and, upon inspection of the results, it is clear (even with the small size of the problem) that the result of the first partitioner is somewhat reasonable while the quality of the second partition is quite poor. To quantify the partition quality, the number of elements, nodes, and edge-cuts for each processor is summarized in Table I The number of the edgecuts for the "bad" partition is clearly much larger than that

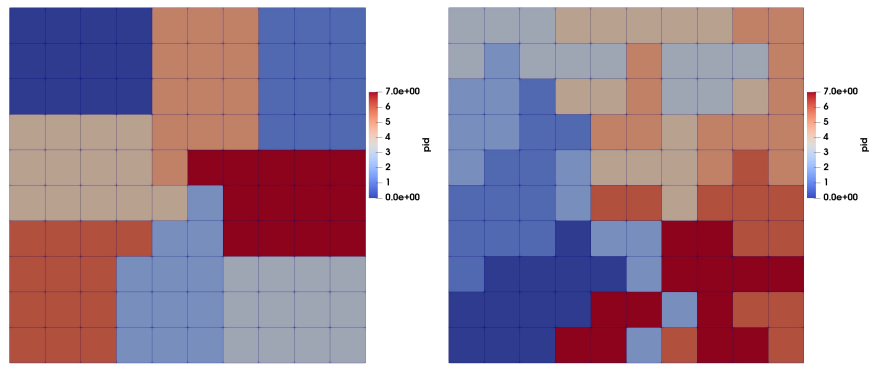

Fig. 1: "Good" (left) and "bad" (right) examples of partitioning onto 8 subdomains. Each color corresponds to a different subdomain.

TABLE I: 2D partitioning example. "pid" is the MPI rank, "elems" is the number of elements, "nodes" is the number of nodes, and "edge-cuts" is the number of edge-cuts.

\begin{tabular}{cccc}
\hline \multicolumn{4}{c}{ Good partition example } \\
\hline pid & elems & nodes & edge-cuts \\
\hline 0 & 12 & 20 & 7 \\
1 & 12 & 20 & 7 \\
2 & 12 & 21 & 13 \\
3 & 12 & 16 & 7 \\
4 & 13 & 14 & 13 \\
5 & 13 & 10 & 13 \\
6 & 13 & 12 & 9 \\
7 & 13 & 8 & 13 \\
\hline
\end{tabular}

\begin{tabular}{cccc}
\hline \multicolumn{4}{c}{ Bad partition example } \\
\hline pid & elems & nodes & edge-cuts \\
\hline 0 & 12 & 22 & 13 \\
1 & 12 & 17 & 17 \\
2 & 13 & 18 & 30 \\
3 & 12 & 21 & 21 \\
4 & 13 & 14 & 29 \\
5 & 13 & 11 & 23 \\
6 & 12 & 15 & 24 \\
7 & 13 & 3 & 23 \\
\hline
\end{tabular}

for the "good" partition, and the workload, as measured by the number of nodes and elements in each partition, is much more evenly split for the "good" partition than it is for the "bad" one.

The basic idea of hierarchical graph partitioning, which has been around for some time (see, e.g., the Zoltan [5] library in Trilinos), is to recursively partition a graph two or more times, possibly onto different numbers of processors each time. For example, the graph may be initially partitioned into $n p_{1}$ subgraphs (where $n p_{1}$ is, say, the number of compute nodes) using an existing partitioner such as ParMETIS, PTScotch, etc. Each subgraph can then be further partitioned into $n p_{2}$ smaller subgraphs (where $n p_{2}$ is e.g. the number of processor cores per compute node), so that the final partition has $n p=n p_{1} \times n p_{2}$ partitions.

The basic idea is simple but very effective, especially when the number of processor cores is large relative to the number of compute nodes, as the likelihood of the algorithm failing increases with the number of partitions asked for in a single iteration. If the number of compute nodes is itself large, the first step of "Hierarch" can be obtained in a recursive manner. For simplicity, only the "two-level" hierarchical partitioning method is discussed in this work. Two hierarchical partitioning examples are shown in Figs. 2 and 3 In these cases, the original meshes are initially partitioned into 2 submeshes, and then each submesh is further split into 4 smaller submeshes. In total, 8 submeshes are obtained through the hierarchical partitioning algorithm, and they can be assigned to processor cores in such a way that adjacent partitions are computed by 


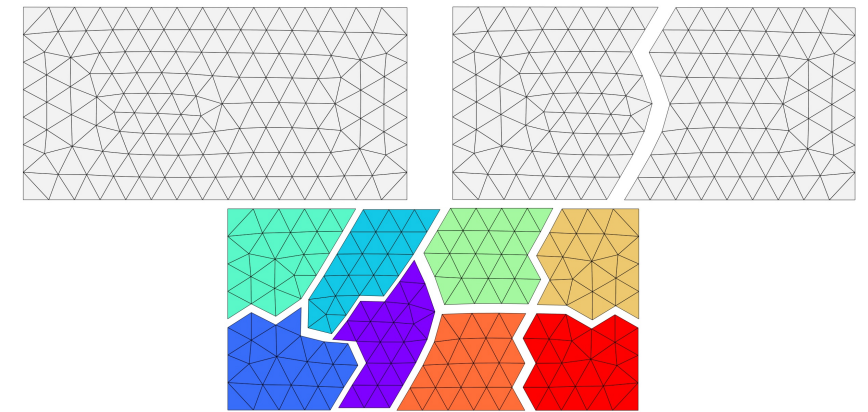

Fig. 2: A 2D hierarchical partitioning example. The original mesh in the top left is partitioned into the two submeshes shown in the top right, and each sub-mesh is further partitioned into the 4 small submeshes shown in the second row.

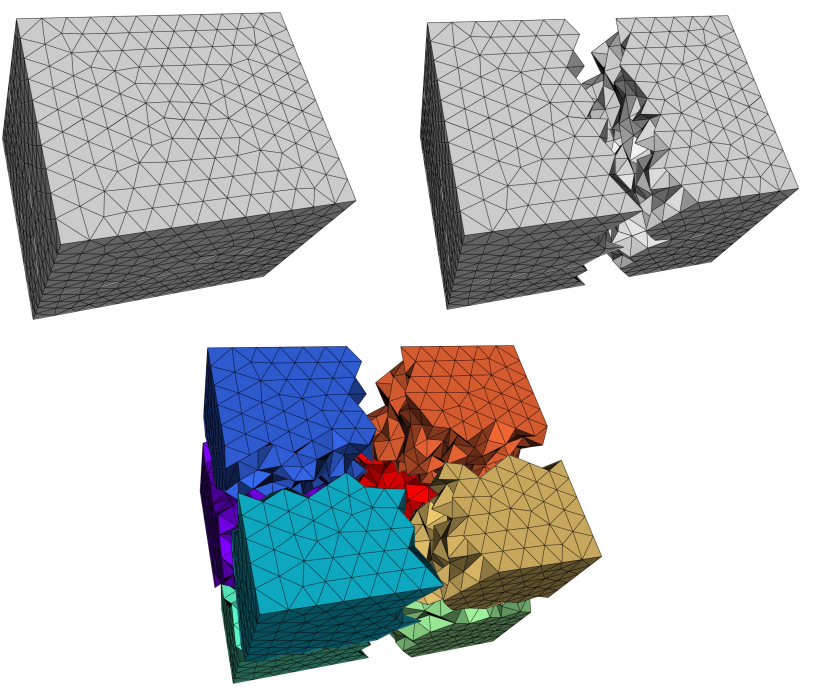

Fig. 3: A 3D hierarchical partitioning 3D example. As in the 2D case, the 3D mesh is partitioned into 2 submeshes at the first step, and then each submesh is subsequently split into 4 smaller submeshes.

processor cores on the same compute node.

To fix ideas and assist in describing the algorithm, let us denote the dual graph of the mesh as $G=\left(\left\{v_{i}\right\},\left\{e_{j}\right\}\right)$, where $v_{i}$ is a graph vertex corresponding to a mesh element, and $e_{j}$ is a graph edge representing a mesh element side. The number of graph vertices $\left\{v_{i}\right\}$ is denoted by $n v$, and a partition is represented by an integer array $P=\left\{p_{i}\right\}, p_{i} \in[0, n p)$, of size $n v$. Vertices $v_{i}$ and $v_{j}$ are assigned to the same partition if and only if $p_{i}=p_{j}$. The number of times $p_{i}$ is repeated indicates how many vertices are assigned to partition $p_{i}$. To carry out the partitioning scheme in parallel, the graph $G$ is assumed to be initially distributed across the processor cores. This can be accomplished by computing e.g. the "trivial" partitioning defined by assigning the first chunk of vertices (ordered by vertex ID) to the first processor, the second chunk to the second processor, and so on.

In the first step of the hierarchical partitioning scheme, the distributed graph $G$ is partitioned by applying an existing algorithm such as ParMETIS or PTScotch to produce a partition $P^{1}=\left\{p_{i}^{1}\right\}, p_{i}^{1} \in\left[0, n p_{1}\right), i=0,1, \ldots, n v-1$. In order to carry out the second partitioning step, $n p_{1}$ subgraphs have to be assembled and allocated to the first $n p_{1}$ processors, with each processor taking one subgraph. If the partitioning process is carried out as a preprocessing step, the number of processor cores used in the second step can be smaller than $n p_{1}$. If this happens, more than one subdomain will be allocated to the same processor core. We do not discuss this situation in the present work since it is straightforward to extend the algorithm to handle it, but our implementation in PETSC does support this use case.

Two steps are required to construct a local graph from $P^{1}$. The information in $P^{1}$ tells us where we should send the vertex IDs, i.e. $v_{i}$ should be sent to the $p_{i}^{1}$ th processor, but the required data can't be exchanged in a single communication step. Instead, communication ranks and data sizes have to be discovered before the vertex IDs can be sent. The twosided information discovery operation is carried out using the algorithm discussed in [19].

After the discovery, each processor knows how much information it will receive and from whom it will receive it. The one-to-many sparse communication pattern is efficiently implemented by the "star forest" communication object in PETSc called PetSCSF [20]. This vertex ID exchange algorithm is summarized and implemented in the ISBuildTwoSided routine in PETSc.

Once the vertex exchange is complete, a vertex set $V_{c}$ is created for the $c$ th processor, where $c \in\left[0, n p_{1}-1\right]$. A subgraph $G_{c}$ is extracted from the global graph $G$ through, once again, a sparse communication. The size of $G_{c}$ is denoted as $n v_{c}$. Finally, a serial partitioner (or parallel partitioner on a single processor) is applied to partition $G_{c}$ to produce $\tilde{P}_{c}^{2}=\left\{p_{c, k}^{2}\right\}, p_{c, k}^{2} \in\left[0, n p_{2}-1\right), k=0,1, \ldots, n v_{c}-1, c=$ $0,1, \ldots, n p_{1}-1$. The $\tilde{P}_{c}^{2}$ are sent back to the original owners, and then are merged based on the global vertex IDs. The new second-step partition is denoted as $P^{2}=\left\{p_{i}^{2}\right\}, p_{i}^{2} \in\left[0, n p_{2}-\right.$ 1), $i=0,1, \ldots, n v-1$, the entries of which are a permutation of $\left\{p_{0,0}^{2}, p_{0,1}^{2}, \ldots, p_{0, n v_{0}-1}^{2}, \ldots, p_{c, 0}^{2}, p_{c, 1}^{2}, \ldots, p_{c, n v_{c}-1}^{2}, \ldots\right\}$. The final partition $P$ is defined as follows:

$$
P=\left\{p_{i}=p_{i}^{1} \times n p_{2}+p_{i}^{2}\right\}, p_{i}^{1} \in P^{1}, p_{i}^{2} \in P^{2} .
$$

The entire process is summarized in Algorithm 1, where we assume $G$ is initially distributed across the processors.

For some applications we need an arbitrary number, $n p$, of subgraphs, but $n p$ cannot be factored into $n p_{1} \times n p_{2}$. Algorithm 1 has to be adjusted to handle this situation, and our basic approach is to apply subdomain weights in the first step so that a subgraph may have a different size from other subgraphs, depending on how many smaller subgraphs it will be further divided into. The remainder $r=n p \bmod n p_{2}$ is computed for a user-specified $n p_{2}$ (usually $n p_{2}$ is the number of processor cores per compute node), and $n p_{1}$ is calculated 


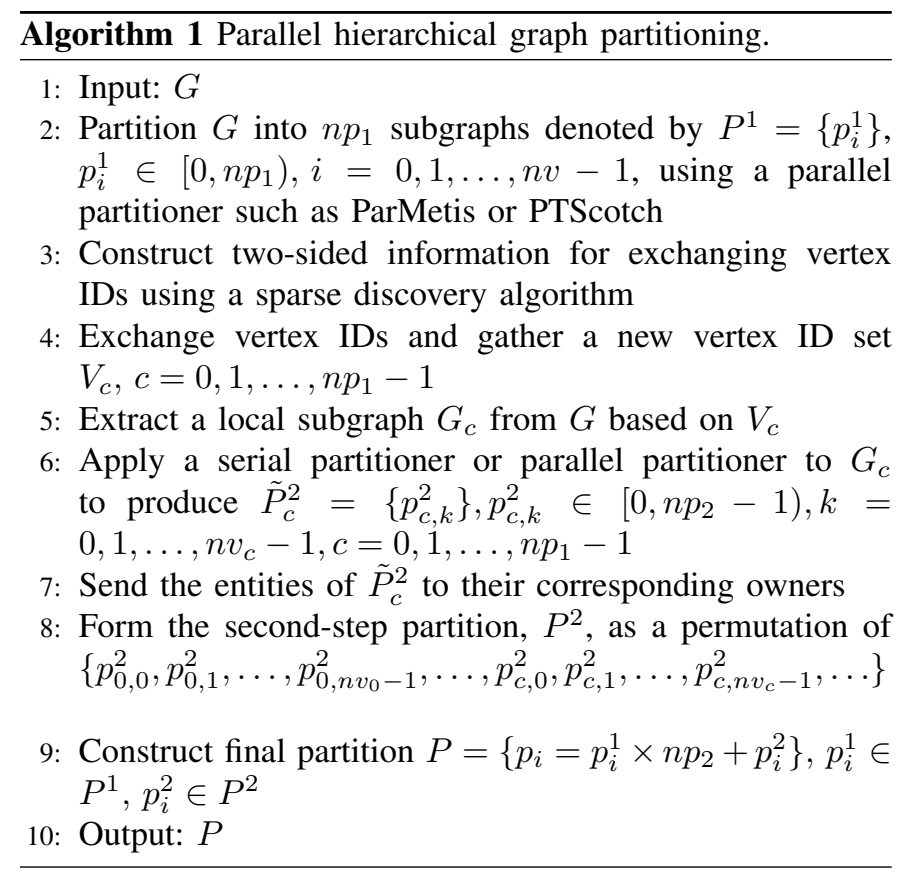

according to:

$$
n p_{1}=\left\{\begin{aligned}
n p / n p_{2}, & \text { if } r=0 \\
n p / n p_{2}+1, & \text { otherwise. }
\end{aligned}\right.
$$

The number of small subgraphs for each subgraph, $S=$ $\left\{s_{c}\right\}, c=0,1, \ldots, n p_{1}-1$, is constructed as follows:

$$
s_{c}=\left\{\begin{aligned}
r, & \text { if } c=0 \text { and } r \neq 0 \\
n p_{2}, & \text { otherwise. }
\end{aligned}\right.
$$

Subdomain weights are simply derived from $S$ as $W=$ $w_{c}=s_{c} / n p, c=0,1, \ldots, n p_{1}-1$. A smaller weight indicates that less vertices are assigned to the corresponding subgraph. An offset array, $O=\left\{o_{c}\right\}, c=0,1, \ldots, n p_{1}-1$, of size $n p_{1}$ is needed for forming the final partition, and it is constructed by accumulating the entities of $S$, that is,

$$
o_{c}=\left\{\begin{aligned}
0, & \text { if } c=0, \\
s_{c}+o_{c-1}, & \text { otherwise. }
\end{aligned}\right.
$$

In the second phase of the hierarchical partitioning, the subgraph $G_{c}$ is partitioned into $s_{c}$ small subgraphs, and the corresponding partition is denoted as $\tilde{P}_{c}^{2}$. Note that it is different from the previous algorithm where the $G_{c}$ is always partitioned into $n p_{2}$ smaller subgraphs. The second step partition is formed similarly as $P^{2}$. The final partition, $P=\left\{p_{i}\right\}$, is formed as

$$
p_{i}=o_{p_{i}^{1}}+p_{i}^{2}, p_{i}^{1} \in P^{1}, p_{i}^{2} \in P^{2} .
$$

The generalized parallel hierarchical graph partitioning procedure is summarized in Algorithm 2. The algorithm was implemented in PETSC [21] as part of this work, and can be accessed through the command-line option: -mat_partitioning_type hierarch.

Fig. 4 illustrates the process of partitioning a mesh into $n p$ processors when $n p$ and $n p_{2}$, the number of processor cores on $\overline{\text { Algorithm } 2 \text { General-purpose parallel hierarchical graph par- }}$ titioning.

1: Input: $G$

2: Compute $W=\left\{w_{c}\right\}, O=\left\{o_{c}\right\}$ and $S=\left\{s_{c}\right\}$

3: Partition $G$ into $n p_{1}$ subgraphs denoted by $P^{1}=\left\{p_{i}^{1}\right\}$, $p_{i}^{1} \in\left[0, n p_{1}\right), i=0,1, \ldots, n v-1$, using a parallel partitioner such as ParMetis or PTScotch together with $W$

4: Construct two-sided information for exchanging vertex IDs using a sparse discovery algorithm

5: Exchange vertex IDs and gather a new vertex ID set $V_{c}, c=0,1, \ldots, n p_{1}-1$

6: Extract a local subgraph $G_{c}$ from $G$ based on $V_{c}$

7: Partition $G_{c}$ to $s_{c}$ small subgraphs using a serial partitioner or parallel partitioner and then produce $\tilde{P}_{c}^{2}$

8: Send the entities of $\tilde{P}_{c}^{2}$ to their corresponding owners

9: Form second-step partition, $P^{2}$, as a permutation of $\bigcup_{c}^{n p_{1}-1} \tilde{P}_{c}^{2}$

10: Construct final partition $P=\left\{p_{i}=o_{p_{i}^{1}}+p_{i}^{2}\right\}, p_{i}^{1} \in$ $P^{1}, p_{i}^{2} \in P^{2}, i=0,1, \ldots, n v-1$

11: Output: $P$
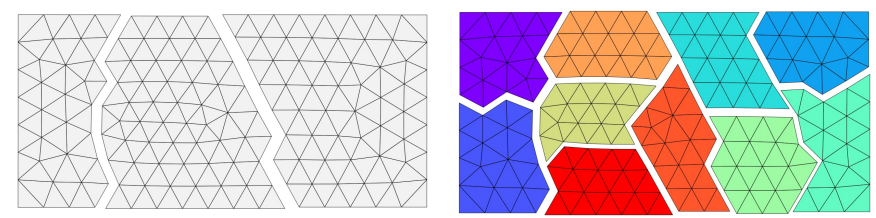

Fig. 4: Demonstration of partitioning a mesh into 10 submeshes. Each compute node has 4 processor cores, and 3 compute nodes are available. The first submesh is split into 2 small submeshes, and the second and third submeshes are partitioned into 4 small submeshes, respectively.

each compute node, are relatively prime. The example assumes that the number of processor cores per compute node is $n p_{2}=$ 4 , but $n p=10$ submeshes are required. In the first step of the algorithm, the mesh is partitioned into 3 subdomains of unequal size, with the first subdomain being relatively smaller than the other two. The first submesh is subsequently split into 2 smaller submeshes while other two submeshes are each divided into 4 smaller submeshes. The resulting 10 partitions are then of nearly equal size.

\section{NODE ASSIGNMENT ALGORITHM}

By default, libMesh [22] assigns mesh nodes at partitioning interfaces to the neighboring MPI process with the lower rank. This simple heuristic can lead to a computational load imbalance in which lower MPI ranks have more mesh nodes than higher MPI ranks, even when the element partition is perfectly balanced.

An alternative, yet still simple and inexpensive, approach for balancing the mesh nodes on each partition is to assign a node randomly to an MPI rank if that rank has at least one of the node's attached elements. Unfortunately, this simple 

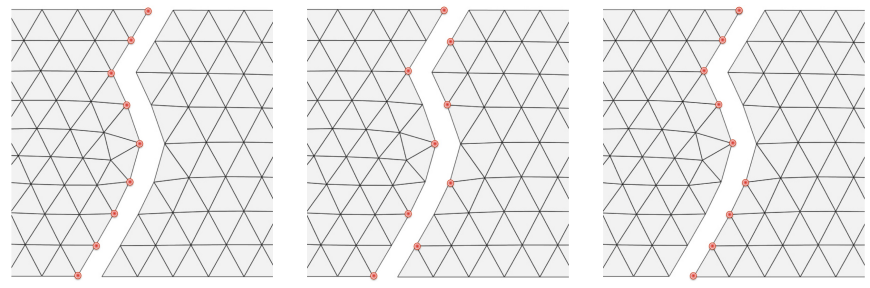

Fig. 5: Different mesh node assignment schemes. Left: shared mesh nodes are assigned to the neighboring MPI process with the lower rank. Middle: mesh nodes with odd IDs are assigned to the lower MPI rank, even IDs are assigned to the higher MPI rank. Right: shared interface nodes are partitioned into two parts using a partitioning algorithm such as ParMetis or PTScotch. The approach on the right is the most efficient for the test case discussed in Sec. IV

approach may cause preconditioner and solver applications to scale unpredictably, and is not optimal for preserving the locality of an element and its neighbor's data structures, which can be detrimental to the efficiency of a finite element solver.

We propose a novel algorithm to resolve this issue. The basic idea is to apply a partitioner to each pair of MPI processes at the lower-dimensional shared interface between processor boundaries, and assign one resulting submesh to each neighboring MPI rank. Note that each interface mesh is shared by only two MPI processes. The basic idea is shown in Fig. 5 in detail. This new mesh node assignment algorithm is implemented in libMesh.

\section{TEST CASE}

In this section, we verify the effectiveness of the proposed algorithms with a test case. The parallel preconditioning efficiency of the proposed hierarchical partitioner is compared to that obtained by applying ParMETIS and PTScotch directly. In particular, the novel node assignment algorithm based on the interface mesh partitioning will be shown to significantly improve preconditioning performance. Due to space constraints, we restrict our testing to a single grain growth example here, but the proposed algorithm also works for other physical applications.

Grain growth is the increase in size of grains in a material due to a reduction of the internal energy that is achieved by reducing the total area of grain boundaries (GBs). GBs migrate to reduce the total free energy of the system. Various sources of free energy drive the GB migration process, including stored defect energy, deformation energy, and GB energy. Various modeling approaches have been applied to model grain boundary migration, and the phase field method has emerged as one of the more popular. In the phase field model, each grain is represented by a continuous order parameter $\eta_{i}$ that is equal to 1 within the grain and equal to 0 in all other grains.
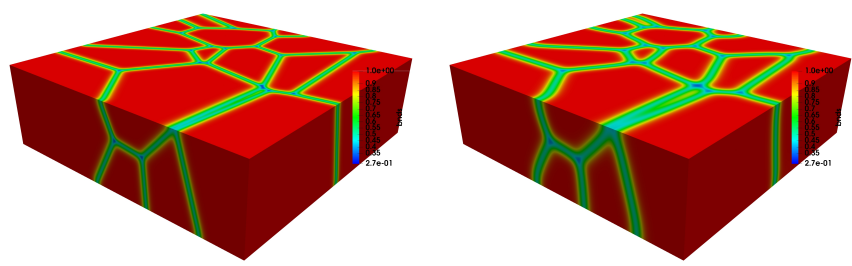

Fig. 6: Solution at $t=25 \mathrm{~ns}$ and $t=250 \mathrm{~ns}$.

The free energy for this problem is

$$
f_{l o c}=\mu\left(\sum_{i}^{N}\left(\frac{\eta_{i}^{4}}{4}-\frac{\eta_{i}^{2}}{2}\right)+\gamma \sum_{i=1}^{N} \sum_{j>i}^{N} \eta_{i}^{2} \eta_{j}^{2}\right)+\frac{1}{4}
$$

where $N$ is the number of order parameters, and $\mu, \gamma$ are material coefficients. The $\eta_{i}$ evolve in space and time according to the Allen-Cahn equation,

$$
\frac{\partial \eta_{j}}{\partial t}=-L_{j} \frac{\delta F}{\delta \eta_{j}}
$$

where $L_{j}$ is the order parameter mobility. Here $F$ is defined as:

$$
F=\int_{V}\left[f_{l o c}\left(\eta_{1}, \ldots, \eta_{N}\right)+f_{g r}\left(\eta_{1}, \ldots, \eta_{N}\right)\right] d V
$$

where the gradient energy density $f_{g r}$ is

$$
f_{g r}=\sum_{j}^{N} \frac{\kappa_{j}}{2}\left|\nabla \eta_{j}\right|^{2} .
$$

The model parameters $L_{j}, \mu$ and $\kappa_{j}$ are defined in terms of the grain boundary (GB) surface energy $\sigma$, the diffuse GB width $w_{G B}$ and the GB mobility $m_{G B}$. The values of the parameters used in the present simulation are: $L_{j}=0.0354524, \mu=$ $0.662848, \gamma=1.5$ and $\kappa_{j}=132.57$.

Eq. (2) is discretized in 3D with 25 grains and 9 order parameters using a first order Lagrange finite element method in MOOSE [23]. (The order parameters are reused for more than one grain based on a coloring of the adjacency matrix representing the grain connection of the microstructures.) The resulting nonlinear system is solved using a Jacobianfree Newton-Krylov method [24], employing GMRES [25] together with a restricted additive Schwarz preconditioner [26], [27]. The impact of the various mesh partitioning methods on the preconditioning efficiency are reported below. For reference, the solution at $t=25 \mathrm{~ns}$ and $250 \mathrm{~ns}$ is shown in Fig. 6

We initially solve this problem on a relatively "coarse" mesh with 9,830,400 hexahedral elements, 9,994,977 nodes and $89,954,793$ unknowns. The preconditioner performance for 10 time steps obtained using various partitioning schemes is reported in Table II The columns of the table are defined as follows: $n p$ is the number of processor cores, "EPart" denotes the partitioner used for partitioning the mesh, "PCSetup" is the compute time in seconds spent on the preconditioner setup, "PCApply" is the compute time in seconds spent on the 
TABLE II: Partitioner performance on "coarse" mesh with $9,830,400$ elements. The times and parallel efficiencies for setting up and applying the preconditioner are measured individually, and the ratio of maximum to minimum node count is reported in the "NR" column.

\begin{tabular}{ccccccc}
\hline$n p$ & EPart & PCSetup & PCApply & PCSEFF & PCAEFF & NR \\
\hline 8,192 & Hierarch & 19.46 & 8.1 & $100 \%$ & $100 \%$ & 1.35 \\
8,192 & ParMETIS & 22.76 & 10.38 & $86 \%$ & $78 \%$ & 1.57 \\
8,192 & PTScotch & 20.33 & 8.92 & $*$ & $*$ & 1.33 \\
\hline 10,240 & Hierarch & 16.21 & 6.6 & $96 \%$ & $98 \%$ & 1.45 \\
10,240 & ParMETIS & 19.09 & 8.17 & $82 \%$ & $79 \%$ & 1.70 \\
10,240 & PTScotch & $*$ & $*$ & $*$ & $*$ & $*$ \\
\hline 16,384 & Hierarch & 11.02 & 5.14 & $88 \%$ & $79 \%$ & 1.54 \\
16,384 & ParMETIS & 13.2 & 5.88 & $74 \%$ & $69 \%$ & 1.94 \\
16,384 & PTScotch & $*$ & $*$ & $*$ & $*$ & $*$ \\
\hline 24,576 & Hierarch & 8.71 & 3.98 & $74 \%$ & $68 \%$ & 1.68 \\
24,576 & ParMETIS & 10.6 & 4.8 & $61 \%$ & $56 \%$ & 2.04 \\
24,576 & PTScotch & $*$ & $*$ & $*$ & $*$ & $*$ \\
\hline 32,768 & Hierarch & 6.8 & 3.15 & $72 \%$ & $64 \%$ & 1.83 \\
32,768 & ParMETIS & 8.46 & 4.35 & $58 \%$ & $47 \%$ & 1.97 \\
32,768 & PTScotch & $*$ & $*$ & $*$ & $*$ & $*$ \\
\hline
\end{tabular}

application of the preconditioner, "PCSEFF" is the parallel efficiency of the preconditioner setup, and "PCAEFF" is the parallel efficiency of the preconditioner application. "NR" is the ratio of the maximum mesh node count to the minimum mesh node count; "NR=1.0" indicates the problem is perfectly balanced.

The test is conducted for processor counts between 8,192 and 32,768 on the Theta supercomputer at Argonne National Laboratory. Theta is a massively parallel, many-core system with second-generation Intel Xeon Phi processors. Each compute node has a 64-core processor with 16 gigabytes (GB) of high-bandwidth in-package memory (MCDRAM), 192 GB of DDR4 RAM, and a 128 GB SSD.

As shown in Table II in the 8,192-core case, PTScotch gives a result similar to the hierarchical partitioning method, but it fails to partition the mesh for all other processor counts, as indicated in the table with an asterisk. ParMETIS is able to generate partitions for all cases, but the partition quality is not as high as Hierarch's in the different metrics reported.

The partition generated using Hierarch has better load balance, e.g. Hierarch's "NR" is 1.45 when using 10,240 processor cores while that of ParMETIS is 1.7. The preconditioner application and setup times are also shorter for Hierarch than for ParMETIS, despite the fact that the same preconditioning algorithm was used in both cases. The parallel efficiencies "PCSEFF" and "PCAEFF" for Hierarch are also 10-20 percentage points higher than the ParMETIS efficiencies, which indicates the proposed algorithm scales better. The efficiencies and corresponding speedup are plotted vs. core count in Fig. 7 .

The results shown in Table II include the new partitionerbased node assignment scheme discussed previously. In order to understand and isolate the importance of the node assignment scheme to the overall preconditioner efficiency, we again solve the "coarse" problem with the hierarchical partitioner, but this time we compare the "default" (minimum MPI rank)
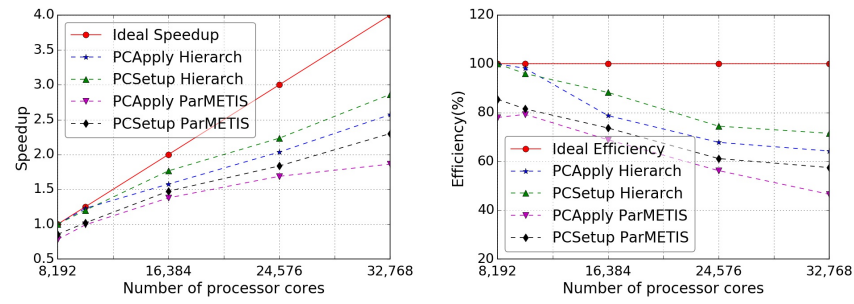

Fig. 7: Speedup and parallel efficiency for various partitioning approaches vs. core count on the "coarse" mesh problem.

TABLE III: Effect of different node assignment algorithms. The "coarse" mesh problem is again solved using the hierarchical partitioner, but in this case we compare the "default" node assignment algorithm (minimum MPI rank) to the interface partitioner approach ("part") for assigning node ownership.

\begin{tabular}{ccccccc}
\hline$n p$ & NAS & PCSetup & PCApply & PCSEFF & PCAEFF & NR \\
\hline 8,192 & default & 22.21 & 13.5 & $88 \%$ & $60 \%$ & 2.35 \\
8,192 & part & 19.46 & 8.1 & $100 \%$ & $100 \%$ & 1.35 \\
\hline 10,240 & default & 20.03 & 13.105 & $78 \%$ & $49 \%$ & 2.72 \\
10,240 & part & 16.21 & 6.6 & $96 \%$ & $98 \%$ & 1.45 \\
\hline 16,384 & default & 13.01 & 8.6 & $75 \%$ & $47 \%$ & 3.04 \\
16,384 & part & 11.02 & 5.14 & $88 \%$ & $79 \%$ & 1.54 \\
\hline 24,576 & default & 9.4 & 6.9 & $69 \%$ & $39 \%$ & 3.76 \\
24,576 & part & 8.71 & 3.98 & $74 \%$ & $68 \%$ & 1.68 \\
\hline 32,768 & default & 8.37 & 5.78 & $58 \%$ & $35 \%$ & 4.1 \\
32,768 & part & 6.8 & 3.15 & $71 \%$ & $64 \%$ & 1.83 \\
\hline
\end{tabular}

and partitioner-based node assignment ("part") schemes.

The numerical results are summarized in Table III and it is easily observed that, in all cases, the new node assignment scheme significantly improves the workload balance and reduces the total compute time. For example, in the 8,192-core case, "NR" is 2.35 when using the default node assignment scheme, while it is reduced to 1.35 when using the partitionerbased node assignment algorithm. Parallel efficiency is generally improved by around $30 \%$ when using the new node assignment algorithm in place of the default one.

To further understand the scalability of the new partitioner, we also ran the same problem on a "fine" mesh with 78,643,200 hexahedral elements and 79,300,033 nodes.
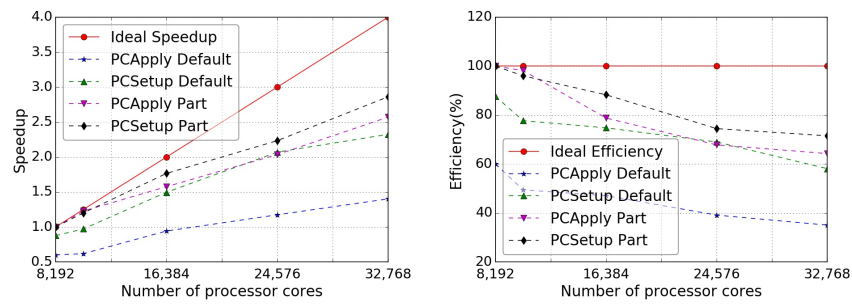

Fig. 8: Speedup and parallel efficiency for different node assignment algorithms vs. core count on the "coarse" mesh problem. 
TABLE IV: Partitioner performance on "fine" mesh with $78,643,200$ elements. PTScotch is omitted because it was not able to successfully generate any partitions of the fine mesh.

\begin{tabular}{ccccccc}
\hline$n p$ & EPart & PCSetup & PCApply & PCSEFF & PCAEFF & NR \\
\hline 8,192 & Hierarch & 134.59 & 80.65 & $100 \%$ & $100 \%$ & 1.14 \\
8,192 & ParMETIS & 138.69 & 98.28 & $97 \%$ & $82 \%$ & 2.4 \\
\hline 10,240 & Hierarch & 115.63 & 71.494 & $93 \%$ & $90 \%$ & 1.16 \\
10,240 & ParMETIS & 121.50 & 78.499 & $88 \%$ & $82 \%$ & 1.87 \\
\hline 16,384 & Hierarch & 78.59 & 41.905 & $86 \%$ & $96 \%$ & 1.22 \\
16,384 & ParMETIS & 85.610 & 68.675 & $79 \%$ & $59 \%$ & 1.64 \\
\hline 24,576 & Hierarch & 56.463 & 29.589 & $79 \%$ & $90 \%$ & 1.24 \\
24,576 & ParMETIS & 65.17 & 41.857 & $69 \%$ & $64 \%$ & 3.2 \\
\hline 32,768 & Hierarch & 47.99 & 20.717 & $70 \%$ & $97 \%$ & 1.34 \\
32,768 & ParMETIS & 54.165 & 34.801 & $62 \%$ & $58 \%$ & 1.79 \\
\hline
\end{tabular}
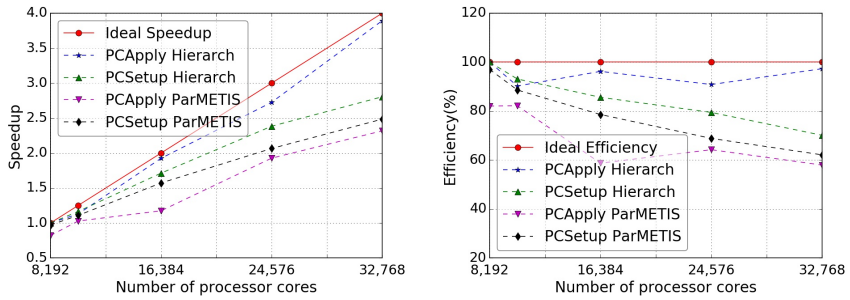

Fig. 9: Speedup and parallel efficiency for various partitioning approaches vs. core count on the "fine" mesh problem.

The resulting system has 713,700,297 unknowns, and is again solved by the Jacobian-free Newton-Krylov method, employing GMRES and a Schwarz preconditioner. Timings and parallel efficiencies for the "fine" mesh are reported in Table IV, where it is once again observed that the hierarchical partitioner improves the preconditioner performance significantly, as compared to ParMETIS. The PTScotch results are omitted in this case since that partitioner was unable to generate a partition of the fine mesh in any of the cases tested.

For the 8,192-core case, "NR" is only 1.14 (close to a perfect balance ratio) when using Hierarch, while it is 2.4 when using ParMETIS. The preconditioning process is $20 \%$ faster for Hierarch than it is for ParMETIS. The ParMETIS partitioner's performance can also be irregular, as observed in the 24,576-core case where "NR" is 3.2 for ParMETIS and only 1.24 for Hierarch. This imbalance leads to much slower preconditioner application and setup times (12s and $10 \mathrm{~s}$, respectively) for the ParMETIS partitioner.

The corresponding parallel efficiencies have a similar pattern, that is, the parallel efficiency of the preconditioner application for Hierarch is $30 \%$ higher than that of ParMETIS, and the parallel efficiency of the preconditioner setup for Hierarch is $10 \%$ higher than that of ParMETIS. The speedup and parallel efficiency for the "fine" mesh case are also summarized in Fig. 9

Similarly, we compare the preconditioning performance for the new node assignment approach with using the "default" node assignment heuristic. We observe that, especially when the number of processor cores is large, it is essential to
TABLE V: Effect of different node assignment strategies for the "fine" mesh. The slightly superlinear PCAEFF value in the 10,240 core case is sometimes observed at smaller core counts depending on the partitioner, but does not signify a general trend.

\begin{tabular}{ccccccc}
\hline$n p$ & NAS & PCSetup & PCApply & PCSEFF & PCAEFF & NR \\
\hline 8,192 & default & 146.42 & 89.844 & $92 \%$ & $90 \%$ & 1.51 \\
8,192 & part & 134.59 & 80.65 & $100 \%$ & $100 \%$ & 1.14 \\
\hline 10,240 & default & 117.35 & 62.092 & $92 \%$ & $104 \%$ & 1.56 \\
10,240 & part & 115.63 & 71.494 & $93 \%$ & $90 \%$ & 1.16 \\
\hline 16,384 & default & 83.736 & 49.294 & $80 \%$ & $82 \%$ & 1.72 \\
16,384 & part & 78.59 & 41.905 & $86 \%$ & $96 \%$ & 1.22 \\
\hline 24,576 & default & 62.149 & 36.264 & $72 \%$ & $74 \%$ & 1.89 \\
24,576 & part & 56.463 & 29.589 & $79 \%$ & $91 \%$ & 1.24 \\
\hline 32,768 & default & 52.769 & 29.520 & $64 \%$ & $68 \%$ & 2.02 \\
32,768 & part & 47.99 & 20.717 & $70 \%$ & $97 \%$ & 1.34 \\
\hline
\end{tabular}
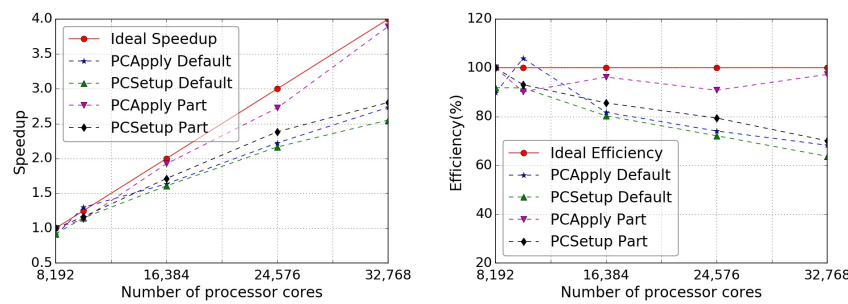

Fig. 10: Speedup and parallel efficiency for different node assignment algorithms vs. core count on the "fine" mesh problem.

maintain a workload balance in order to achieve good parallel efficiency. For example, when we use 32,768 processor cores, "NR" is 2.02 for the default node assignment, while it is 1.34 for the partitioning-based node assignment, and these different balance ratios lead to significantly different overall performance levels. The parallel efficiency of the preconditioner application for the partitioner-based node assignment is $20 \%$ higher than that of the default node assignment algorithm, while the parallel efficiency of the preconditioner setup is about $10 \%$ better. The parallel efficiency and the speedup for the different node assignment algorithms are also summarized in Fig. 10 .

\section{CONCLUSIONS AND FUTURE WORK}

A general-purpose hierarchical mesh partitioning method was introduced and discussed for large-scale scientific computing. The partitioner distributes graphs recursively onto both $n p_{1}$ (the number of compute nodes) and $n p_{2}$ (usually $\leq$ the number of processor cores per compute node) subdomains. Note that $n p_{2}$ can be different on each compute node, making the hierarchical partitioning algorithm useful for general calculations on heterogeneous collections of nodes.

Mesh nodes on inter-processor interfaces are often assigned to the lower MPI rank by default, and this simple choice can lead to a significant load imbalance. The issue is addressed by introducing a new node balancing algorithm in which a 
graph corresponding to the interface mesh shared by two processor cores is partitioned into two submeshes using a partitioner, and one submesh is assigned to the lower MPI rank while the other is sent to the higher MPI rank. This scheme preserves the data locality and maintains a balance in the computational workload. We numerically demonstrate that the hierarchical partitioner approach combined with the new node balancing technique reduces the application and setup time of the preconditioner by about $50 \%$ compared to direct application of existing algorithms.

While this study focused on the correlation between partition quality and preconditioner efficiency, future research will investigate the performance and scalability of other parts of the simulation, including the partitioning process itself.

\section{ACKNOWLEDGMENTS}

This research was supported through the INL Laboratory Directed Research \& Development (LDRD) Program under DOE Idaho Operation Office Contract DE-AC07-05ID14517 and used resources of the Argonne Leadership Computing Facility, which is a DOE Office of Science User Facility supported under Contract DE-AC02-06CH11357. This manuscript has been authored by Battelle Energy Alliance, LLC under Contract No. DE-AC07-05ID14517 with the U.S. Department of Energy. The United States Government retains and the publisher, by accepting the article for publication, acknowledges that the United States Government retains a nonexclusive, paid-up, irrevocable, and worldwide license to publish or reproduce the published form of this manuscript, or allow others to do so, for United States Government purposes.

\section{REFERENCES}

[1] K. Andreev and H. Racke, "Balanced graph partitioning," Theory of Computing Systems, vol. 39, no. 6, pp. 929-939, Nov. 2006, https://doi. org/10.1007/s00224-006-1350-7

[2] A. E. Feldmann and L. Foschini, "Balanced partitions of trees and applications," Algorithmica, vol. 71, no. 2, pp. 354-376, Feb. 2015, https://doi.org/10.1007/s00453-013-9802-3

[3] B. Nour-Omid, A. Raefsky, and G. Lyzenga, "Solving finite element equations on concurrent computers," in Proceedings of Parallel Computations and Their Impact on Mechanics, Boston, Massachusetts, USA, Dec. 13-18, 1987.

[4] C. Farhat, "A simple and efficient automatic FEM domain decomposer," Computers \& Structures, vol. 28, no. 5, pp. 579-602, 1988, https://doi. org/10.1016/0045-7949(88)90004-1

[5] K. Devine, E. Boman, R. Heaphy, B. Hendrickson, and C. Vaughan, "Zoltan data management services for parallel dynamic applications," Computing in Science \& Engineering, vol. 4, no. 2, pp. 90-97, Apr. 2002, https://doi.org/10.1109/5992.988653

[6] S. T. Barnard and H. D. Simon, "Fast multilevel implementation of recursive spectral bisection for partitioning unstructured problems," Concurrency: Practice and Experience, vol. 6, no. 2, pp. 101-117, Apr. 1994, https://doi.org/10.1002/cpe.4330060203

[7] G. Karypis and V. Kumar, "A fast and high quality multilevel scheme for partitioning irregular graphs," SIAM Journal on Scientific Computing, vol. 20, no. 1, pp. 359-392, 1998, https://doi.org/10.1137/ S1064827595287997

[8] B. W. Kernighan and S. Lin, "An efficient heuristic procedure for partitioning graphs," The Bell System Technical Journal, vol. 49, no. 2, pp. 291-307, Feb. 1970, https://doi.org/10.1002/j.1538-7305.1970.tb01770. X
[9] C. M. Fiduccia and R. M. Mattheyses, "A linear-time heuristic for improving network partitions," in Proceedings of the 19th Design Automation Conference, Piscataway, New Jersey, USA, 1982, pp. 175181, https://dl.acm.org/citation.cfm?id=809204

[10] B. Hendrickson and R. Leland, "Chaco: Algorithms and Software for Partitioning Meshes," Sandia National Laboratories, Tech. Rep., 1995, http://www.cs.sandia.gov/CRF/chac_p2.html

[11] G. Karypis and V. Kumar, "METIS-Unstructured graph partitioning and sparse matrix ordering system," University of Minnesota, Department of Computer Science, Tech. Rep., Aug. 1995.

[12] C. Chevalier and F. Pellegrini, "PT-Scotch: A tool for efficient parallel graph ordering," Parallel Computing, vol. 34, no. 6-8, pp. 318-331, Jul. 2008, https://doi.org/10.1016/j.parco.2007.12.001

[13] G. Karypis and K. Schloegel, "ParMETIS-Parallel graph partitioning and sparse matrix ordering library, version 4.0," University of Minnesota, Tech. Rep., 2013, http://glaros.dtc.umn.edu/gkhome/metis/ parmetis/overview

[14] F. Kong and X.-C. Cai, "Scalability study of an implicit solver for coupled fluid-structure interaction problems on unstructured meshes in 3D," The International Journal of High Performance Computing Applications, vol. 32, no. 2, pp. 207-219, 2018, https://doi.org/10.1177\% 2F1094342016646437

[15] _ - "A highly scalable multilevel schwarz method with boundary geometry preserving coarse spaces for $3 \mathrm{~d}$ elasticity problems on domains with complex geometry," SIAM Journal on Scientific Computing, vol. 38, no. 2, pp. C73-C95, 2016, https://doi.org/10.1137/15M1010567

[16] _ _A scalable nonlinear fluid-structure interaction solver based on a schwarz preconditioner with isogeometric unstructured coarse spaces in 3d," Journal of Computational Physics, vol. 340, pp. 498-518, Jul. 2017, https://doi.org/10.1016/j.jcp.2017.03.043

[17] F. Kong, V. Kheyfets, E. Finol, and X.-C. Cai, "An efficient parallel simulation of unsteady blood flows in patient-specific pulmonary artery," International Journal for Numerical Methods in Biomedical Engineering, vol. 34, no. 4, pp. 29-52, Apr. 2018, https://doi.org/10.1002/cnm.2952

[18] F. Kong, "A Parallel Implicit Fluid-structure Interaction Solver with Isogeometric Coarse Spaces for 3D Unstructured Mesh Problems with Complex Geometry," Ph.D. dissertation, University of Colorado Boulder, 2016.

[19] T. Hoefler, C. Siebert, and A. Lumsdaine, "Scalable communication protocols for dynamic sparse data exchange," in Proceedings of the 15th ACM SIGPLAN Symposium on Principles and Practice of Parallel Programming (PPoPP'10), Bangalore, India, Jan. 9-14, 2010, pp. 159168, https://doi.org/10.1145/1693453.1693476

[20] J. Brown, "Star forests as a parallel communication model," Argonne National Laboratory, Tech. Rep., 2011, http://lists.mcs.anl.gov/pipermail/ petsc-dev/attachments/20111224/7bfale70/attachment.pdf

[21] S. Balay, S. Abhyankar, M. F. Adams, J. Brown, P. Brune, K. Buschelman, L. Dalcin, V. Eijkhout, W. D. Gropp, D. Kaushik, M. G. Knepley, D. A. May, L. C. McInnes, R. T. Mills, T. Munson, K. Rupp, P. Sanan, B. F. Smith, S. Zampini, H. Zhang, and H. Zhang, "PETSc users manual," Argonne National Laboratory, Tech. Rep. ANL-95/11, Revision 3.9, 2018, http://www.mcs.anl.gov/petsc

[22] B. S. Kirk, J. W. Peterson, R. H. Stogner, and G. F. Carey, "libMesh: A C++ Library for Parallel Adaptive Mesh Refinement/Coarsening Simulations," Engineering with Computers, vol. 22, no. 3-4, pp. 237254, 2006, https://doi.org/10.1007/s00366-006-0049-3

[23] D. R. Gaston, C. J. Permann, J. W. Peterson, A. E. Slaughter, D. Andrš, Y. Wang, M. P. Short, D. M. Perez, M. R. Tonks, J. Ortensi, L. Zou, and R. C. Martineau, "Physics-based multiscale coupling for full core nuclear reactor simulation," Annals of Nuclear Energy, Special Issue on Multi-Physics Modelling of LWR Static and Transient Behaviour, vol. 84, pp. 45-54, Oct. 2015, https://doi.org/10.1016/j.anucene.2014.09.060

[24] D. A. Knoll and D. E. Keyes, "Jacobian-free Newton-Krylov methods: A survey of approaches and applications," Journal of Computational Physics, vol. 193, no. 2, pp. 357-397, Jan. 2004, https://doi.org/10.1016/ j.jcp.2003.08.010

[25] Y. Saad and M. H. Schultz, "GMRES: A generalized minimal residual algorithm for solving nonsymmetric linear systems," SIAM Journal on Scientific and Statistical Computing, vol. 7, no. 3, pp. 856-869, 1986, https://doi.org/10.1137/0907058

[26] X.-C. Cai and M. Sarkis, "A restricted additive Schwarz preconditioner for general sparse linear systems," SIAM Journal on Scientific Computing, vol. 21, no. 2, pp. 792-797, 1999, https://doi.org/10.1137/ S106482759732678X 
[27] F. Kong, Y. Wang, S. Schunert, J. W. Peterson, C. J. Permann, D. Andrš, and R. C. Martineau, "A fully coupled two-level Schwarz preconditioner based on smoothed aggregation for the transient multigroup neutron diffusion equations," Numerical Linear Algebra with Applications, vol. 25 , no. 3, pp. 21-62, 2018, https://doi.org/10.1002/nla.2162 